\title{
A Barrier Version of the Russian Option
}

\author{
L. A. Shepp, A. N. Shiryaev, A. Sulem \\ Rutgers University; shepp@stat.rutgers.edu \\ Steklov Mathematical Institute; shiryaev@mi.ras.ru \\ INRIA- Rocquencourt; agnes.sulem@inria.fr
}

March 5, 2002

\begin{abstract}
For geometrical Brownian motion we consider the problem of finding the optimal stopping time and the value function for a Russion (put) option, assuming that the decision about stopping should be taken before the process of prices reaches "dangerous" a barrier on the level $\varepsilon>0$.
\end{abstract}

\section{Introduction}

1. In the stochastic financial mathematics there are two "classical" models which form a basis for description of dynamics of the financial indexes (say, stocks).

The first one, the Bachelier model, assumes that the stocks prices $X=$ $\left(X_{t}\right)_{t \geq 0}$ are described as follows:

$$
X_{t}=x+\mu t+\sigma B_{t}
$$

where $x, \mu \in R, \sigma \in R_{+}$and $B=\left(B_{t}\right)_{t \geq 0}$ is a standard Brownian motion.

The second one, more realistic, (Samuelson, Black, Merton, Scholes) assumes that the process of prices $X=\left(X_{t}\right)_{t \geq 0}$ is a geometrical (economical) Brownian motion:

$$
X_{t}=x \exp \left\{\left(\mu-\frac{1}{2} \sigma^{2}\right) t+\sigma B_{t}\right\}
$$


By Ito's formula

$$
X_{t}=x+\int_{0}^{t} \mu X_{s} d s+\int_{0}^{t} \sigma X_{s} d B_{s}
$$

or, symbolically,

$$
d X_{t}=X_{t}\left(\mu d t+\sigma d B_{t}\right), \quad X_{0}=x .
$$

Note that in the Bachelier model (1) the values $X_{t} \in \mathbf{R}$, but in the more realistic model (2) the values of $X_{t} \in \mathbf{R}_{+}$.

2. In [1]-[3], some problems were considered in connection with options of American type. These papers give the explicit formulae for fair (rational) price of the "Russian options" (put and call) and also expressions for the optimal time of the decision about buying or selling the corresponding options.

For the Bachelier model in the case of the put option the problem is to find the fair (rational) price

$$
V^{*}(x)=\sup _{\tau \in \mathcal{M}} \mathbf{E}\left[\max _{u \leq \tau} X_{u}-c \tau\right],
$$

where $\mathcal{M}$ is the class of all stopping times with respect to the Brownian filtration $\left(\mathcal{F}_{t}^{B}\right)_{t \geq 0}, \mathcal{F}_{t}^{B}=\sigma\left(B_{s}, s \leq t\right)$, and to find also the corresponding optimal stopping time $\tau^{*}$.

The financial meaning of the expression " $\max _{u \leq \tau} X_{u}-c \tau$ " is clear: if the buyer of the option takes a decision at time $\tau$, then he obtains the maximum of the values of $X_{u}$ on the time interval $[0, \tau]$ (that is, $\max _{u \leq \tau} X_{u}$ ) taking into account a linear inflation $(-c \tau)$.

The problem dual to (5) is to find the fair (rational) price of the call option

$$
V_{*}(x)=\inf _{\tau \in \mathcal{M}} \mathbf{E}\left[\min _{u \leq \tau} X_{u}+c \tau\right]
$$

and the corresponding optimal stopping time $\tau_{*}$.

An interpretation of the expression " $\min _{u \leq \tau} X_{u}+c \tau$ " is also clear: the buyer has a right to buy the stock at minimum past price $\left(\min _{u \leq \tau} X_{u}\right)$ but with linear discount $(c \tau)$.

Let us indicate the connection between formulations (5) and (6) and the standard $(X-K)^{+}$- and $(K-X)^{+}$-formulations. 
Consider the American call option with payoff function

$$
f_{\tau}=\left(X_{\tau}-K_{*}(X, \tau)\right)^{+}
$$

where $K_{*}(X, \tau)=\min _{u \leq \tau} X_{u}+r \tau$ ("minimum price in discounted dollars"). If $\tau$ is such that $X_{\tau}-K_{*}(X, \tau) \geq 0$ then (if $\mathbf{E} B_{\tau}=0$ )

$$
\begin{aligned}
\sup _{\tau} \mathbf{E}\left(X_{\tau}-K_{*}(X, \tau)\right)^{+} & =\sup _{\tau} \mathbf{E}\left(X_{\tau}-K_{*}(X, \tau)\right) \\
& =x+\sup _{\tau} \mathbf{E}\left[\sigma B_{\tau}+\mu \tau-\min _{u \leq \tau} X_{u}-r \tau\right] \\
& =x+\sup _{\tau} \mathbf{E}\left[(\mu-r) \tau-\min _{u \leq \tau} X_{u}\right] \\
& =x-\inf _{\tau} \mathbf{E}\left[\min _{u \leq \tau} X_{u}+c \tau\right],
\end{aligned}
$$

where $c=r-\mu(r>0$ if and only if $c>-\mu)$.

Similarly, for a put option with $K^{*}(X, \tau)=\max _{u \leq \tau} X_{u}-r \tau$ we find that

$$
\left.\sup _{\tau}\left(K^{*}(X, \tau)-X_{\tau}\right)\right)^{+}=x+\sup _{\tau} \mathbf{E}\left[\max _{u \leq \tau} X_{u}-c \tau\right]
$$

where $c=r+\mu(r>0$ if and only if $c>\mu)$.

So, we see that problems (5) and (6) can be rewritten in the standard $(K-X)^{+}$- and $\left.X-K\right)^{+}$-forms. From [1], it follows that if $c>\mu$ then for problem (5) an optimal stopping time is

$$
\tau^{*}=\inf \left\{t: \max _{u \leq t} X_{u}=X_{t}+\theta^{*}\right\}
$$

where $\theta^{*}$ is some positive constant. (For the case $c \leq \mu$ see [1]).

For problem (6) in the case $c>-\mu$ we have from [3] that

$$
\tau_{*}=\inf \left\{t: \min _{u \leq t} X_{u}=X_{t}-\theta_{*}\right\}
$$

where $\theta_{*}$ is a positive constant. (For the case $c \leq-\mu$ see again [3].)

3. Let us denote

$$
T_{a}=\inf \left\{t: X_{t}=a\right\}, \quad a \in R,
$$

and put

$$
V_{\varepsilon}^{*}(x)=\sup _{\tau \in \mathcal{M}_{\varepsilon}^{*}} \mathbf{E}\left[\max _{u \leq \tau} X_{u}-c \tau\right],
$$


where

$$
\mathcal{M}_{\varepsilon}^{*}=\left\{\tau \in \mathcal{M}: \tau \leq T_{-\varepsilon}\right\}
$$

Correspondingly, denote

$$
V_{*}^{\varepsilon}(x)=\inf _{\tau \in \mathcal{M}_{*}^{\varepsilon}} \mathbf{E}\left[\min _{u \leq \tau} X_{u}+c \tau\right],
$$

where

$$
\mathcal{M}_{*}^{\varepsilon}=\left\{\tau \in \mathcal{M}: \tau \leq T_{\varepsilon}\right\}
$$

Comparing (5) and (10) we see that if in (5) there is no restrictions on the class of admissible stopping times $\tau$, in the case of (10) we admit only stopping times $\tau \in \mathcal{M}_{\varepsilon}^{*}$ which are less or equal to the time of the first reach of the barrier on the level $-\varepsilon$, that is, $\tau \leq T_{-\varepsilon}=\inf \left\{t: X_{t}=-\varepsilon\right\}$.

Financial and economical meaning of the problems (10) and (12) is clear. In the case of (10) we must take decision before default time $T_{-\varepsilon}$ after which all economic activity is stopped. Similarly, we may give the corresponding interpretation of problem (12).

We formulate now the structure of the optimal stopping times $\tau_{\varepsilon}^{*}$ and $\tau_{*}^{\varepsilon}$. In the first case,

$$
\tau_{\varepsilon}^{*}=\inf \left\{t: X_{t} \leq g_{\varepsilon}^{*}\left(S_{t}\right)\right\}
$$

where $S_{t}=\max _{u \leq t} X_{u}$ and

$$
g_{\varepsilon}^{*}(s)=\max \left\{-\varepsilon, s-\theta^{*}\right\} .
$$

In the second case,

$$
\tau_{*}^{\varepsilon}=\inf \left\{t: X_{t} \leq g_{*}^{\varepsilon}\left(M_{t}\right)\right\},
$$

where $M_{t}=\min _{u \leq t} X_{t}$ and

$$
g_{*}^{\varepsilon}(m)=\min \left\{\varepsilon, m+\theta_{*}\right\} .
$$

These results can be obtained by the method which we apply below for a more realistic case of the geometrical Brownian motion. Our main aim here is to consider the barrier put version of the Russian option for $\varepsilon>0$. For completeness we shall give also the corresponding results for the case (5), when $\varepsilon=0$. It will make more clear our approach to the corresponding barrier version $(\varepsilon>0)$. 


\section{Russian put option.}

1. We consider a $(R, S)$-market, where a bank account $R=\left(R_{t}\right)_{t \geq 0}$ satisfies

$$
d R_{t}=r R_{t} d t, \quad R_{0}=1
$$

that is, $R_{t}=e^{r t}, t \geq 0$. General Arbitrage theory ([4, Chapters 7 and 8$]$ ) claims that $(R, X)$-market with geometrical Brownian motion $X=\left(X_{t}\right)_{t \geq 0}$, given by (4), is arbitrage free and for finding the rational (fair) price and optimal stopping times we need to make all calculations with respect to the Wiener measure taking $\mu=r$. Let us denote

$$
V^{*}(x, s)=\sup _{\tau \in \mathcal{M}} \mathbf{E}_{x, s} e^{-(\lambda+r) \tau} S_{\tau},
$$

where

$$
S_{t}=\max _{u \leq t} X_{u} \vee s
$$

and $\mathbf{E}_{x, s}$ is the expectation under the assumption $X_{0}=x$ and $S_{0}=s$, $s \geq x>0$. The term $e^{-(\lambda+r) \tau}$ in (14) plays the role of the exponential inflation (see [5], where inflation was linear).

Theorem 2.1 (see [1],[2]). In problem (14), the optimal stopping time is given by

$$
\tau^{*}=\inf \left\{t: X_{t} \leq g^{*}\left(S_{t}\right)\right\}
$$

where $g^{*}(s)=s / \theta^{*}$ with

$$
\theta^{*}=\left(\frac{1-1 / \gamma_{1}}{1-1 / \gamma_{2}}\right)^{1 /\left(\gamma_{2}-\gamma_{1}\right)}
$$

and $\gamma_{1}(<0)$ and $\gamma_{2}(>1)$,

$$
\gamma_{k}=\frac{A}{2}+(-1)^{k} \sqrt{\left(\frac{A}{2}\right)^{2}+B}, \quad k=1,2,
$$

are roots of the equation

$$
\gamma^{2}-A \gamma-B=0
$$




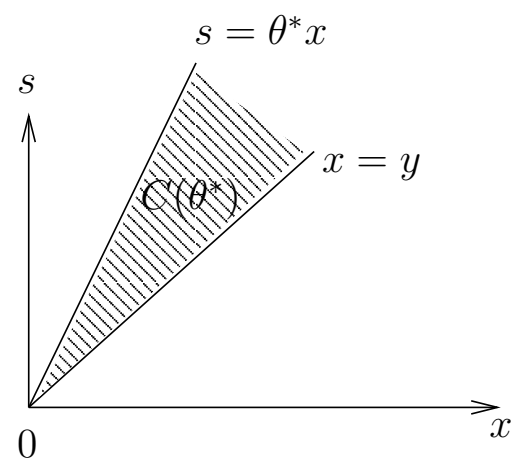

Figure 1: Optimal stopping area and continuation region for the Russian put option

with $A=\frac{1-2 r}{\sigma^{2}}, B=\frac{2(\lambda+r)}{\sigma^{2}}$. Moreover, the value function (14) is given by

$$
V^{*}(x, s)= \begin{cases}\frac{s}{\gamma_{2}-\gamma_{1}}\left[\gamma_{2}\left(\frac{x}{g^{*}(s)}\right)^{\gamma_{1}}-\gamma_{1}\left(\frac{x}{g^{*}(s)}\right)^{\gamma_{2}}\right], & g^{*}(s) \leq x \leq s \\ s, & x \leq g^{*}(s) .\end{cases}
$$

\section{Proof :}

From euristic consideration (and also from the general theory of optimal stopping for Markov processes), we may conclude that one should search optimal stopping in the class of stopping times of the form

$$
\tau(\theta)=\inf \left\{t: X_{t} \leq S_{t} / \theta\right\}
$$

with $\theta>1$. Hence, the problem of finding an optimal stopping time $\tau^{*}$ is reduced to the problem of finding the optimal value $\theta^{*}$ such that

$$
\sup _{\theta>1} \mathbf{E}_{x, s} e^{-(\lambda+r) \tau(\theta)} S_{\tau(\theta)}=\mathbf{E}_{x, s} e^{-(\lambda+r) \tau\left(\theta^{*}\right)} S_{\tau\left(\theta^{*}\right)} .
$$

Note that after finding the value $\theta^{*}$, it is necessary to check that the time $\tau\left(\theta^{*}\right)$ is optimal in the class $\mathcal{M}$ (not only in the class of stopping times $\{\tau(\theta), \theta>1\}$ ). Below we shall do it by using a standard technique of the "verification theorems" based on the Ito-Meyer formula.

Consider the space of states $E=\{(x, s): 0<x \leq s\}$ in which the two-dimensional Markov process $\left(X_{t}, S_{t}\right)_{t \geq 0}$ takes its values. For $\theta>1$ we 
represent $E$ as follows:

$$
E=C(\theta) \cup D(\theta)
$$

where

$$
D(\theta)=\{(x, s) \in E: x \leq s / \theta\}
$$

is the "stopping" area and

$$
C(\theta)=\{(x, s) \in E: s / \theta<x<s\}
$$

is the "continuation" region.

Suppose that $g_{\theta}(s)=s / \theta, s>0$, is a boundary between $C(\theta)$ and $D(\theta)$ (assuming that the points $\left(s, g_{\theta}(s)\right) \in D(\theta)$ ). For search of the optimal $\theta^{*}$ and the value $V^{*}(x, s)$ we consider an auxiliary Stefan (free boundary) problem with unknown functions $g_{\theta}(s)$ and $V=V(x, s)$ :

$$
\begin{gathered}
V(x, s)=s, \quad x \leq g_{\theta}(s), \\
L_{X} V(x, s)=(\lambda+r) V(x, s), \quad g_{\theta}(s)<x<s,
\end{gathered}
$$

with

$$
L_{X}=r x \frac{\partial}{\partial x}+\frac{1}{2} \sigma^{2} x^{2} \frac{\partial^{2}}{\partial x^{2}}
$$

and additional conditions on the boundary $g_{\theta}(s)$ :

$$
\begin{aligned}
& \lim _{x \downarrow g_{\theta}(s)} V(x, s)=s, \\
& \lim _{x \downarrow g_{\theta}(s)} \frac{\partial V(x, s)}{\partial x}=0,
\end{aligned}
$$

and on the diagonal $\{(x, s): 0<x=s\}$ :

$$
\lim _{x \uparrow s} \frac{\partial V(x, s)}{\partial s}=0 .
$$

Condition (21) means that in the stopping area $D(\theta)$ the value $V(x, s)$ should be equal to $s$, which is natural since for $\tau=0$ ("immediate stopping"), 
$\mathbf{E}_{x, s} e^{-(\lambda+r) \tau} S_{\tau}=s$. Condition (22) means that $V(x, s)$ should satisfy the backward Kolmogorov equation (in $x$ ) which is also natural because the functionals $\mathbf{E}_{x, s} e^{-(\lambda+r) \tau(\theta)} S_{\tau(\theta)}$ for $g_{\theta}(s)<x<s$ satisfy (in $x$ ) equation (22). Condition (24) is simply the continuity condition of the function $V(x, s)$ on the boundary (then $x \downarrow g_{\theta}(s)$ ). Condition (25) is the "pasting" or "smooth $f i t$ ' condition. A priori this condition is not very evident, but from an intuitive point of view, it is supported by the idea that the value function should be sufficiently smooth. Finally, condition (26) is a condition of "normal reflection" on the diagonal $\{(x, s) \in E: 0<x=s\}$ that follows from the behaviour of the two-dimensional process $\left(X_{t}, S_{t}\right)_{t \geq 0}$ as a Markov process with reflection.

2. Consider equation (22):

$$
r x \frac{\partial V}{\partial x}+\frac{1}{2} \sigma^{2} x^{2} \frac{\partial^{2} V}{\partial x^{2}}=(\lambda+r) V
$$

for a fixed $s>0$ and $x \in\left(g_{\theta}(s), s\right)$. General solutions of this equation have the form

$$
V(x, s)=B_{1}(s) x^{\gamma_{1}}+B_{2}(s) x^{\gamma_{2}},
$$

where $B_{1}(s)$ and $B_{2}(s)$ are some constants depending on $s$, and $\gamma_{1}$ and $\gamma_{2}$ are defined in (17). From (24), (25) we find that

$$
\begin{aligned}
B_{1}(s)\left(\frac{s}{\theta}\right)^{\gamma_{1}}+B_{2}(s)\left(\frac{s}{\theta}\right)^{\gamma_{2}} & =s, \\
\gamma_{1} B_{1}(s)\left(\frac{s}{\theta}\right)^{\gamma_{1}-1}+\gamma_{2} B_{2}(s)\left(\frac{s}{\theta}\right)^{\gamma_{2}-1} & =0 .
\end{aligned}
$$

and thus

$$
\begin{aligned}
& B_{1}(s)=\frac{s \gamma_{2}}{\gamma_{2}-\gamma_{1}}\left(\frac{\theta}{s}\right)^{\gamma_{1}} \\
& B_{2}(s)=-\frac{s \gamma_{1}}{\gamma_{2}-\gamma_{1}}\left(\frac{\theta}{s}\right)^{\gamma_{2}} .
\end{aligned}
$$

Finally, from (26) under assumption of differentiability of $B_{1}(s)$ and $B_{2}(s)$ we get

$$
B_{1}^{\prime}(s) s^{\gamma_{1}}+B_{2}^{\prime}(s) s^{\gamma_{2}}=0
$$


From (29), (30) and (31) we obtain the values $\theta$ and $V=V(x, s)$ (denote them $\tilde{\theta}$ and $\widetilde{V}(x, s))$ defined by the right-hand sides of (16) and (18).

Now we must prove that the solution of the Stefan problem gives, in fact, the solution to the optimal stopping problem (14), that is, $V^{*}(x, s)=\widetilde{V}(x, s)$. For that it is sufficient to prove that

(a) for each $\tau \in \mathcal{M}$,

$$
\mathbf{E}_{x, s} e^{-(\lambda+r) \tau} S_{\tau} \leq \widetilde{V}(x, s),
$$

(b) for the stopping time

$$
\tilde{\tau}=\inf \left\{t \geq 0: X_{t} \leq g_{\tilde{\theta}}\left(S_{t}\right)\right\}
$$

we have

$$
\mathbf{E}_{x, s} e^{-(\lambda+r) \tilde{\tau}} S_{\tilde{\tau}}=\tilde{V}(x, s)
$$

Indeed,

$$
V^{*}(x, s)=\sup _{\tau \in \mathcal{M}} \mathbf{E}_{x, s} e^{-(\lambda+r) \tau} S_{\tau} \leq \widetilde{V}(x, s),
$$

and because $\mathbf{P}_{x, s}(\tilde{\tau}<\infty)=1$ for all $(x, s) \in E$, then from (34) we get that $\tilde{\tau}$ is an optimal stopping time in $\mathcal{M}$. (From here it is clear, of course, that $\theta^{*}=\tilde{\theta}$ and $g_{\tilde{\theta}}(s)=g_{\theta^{*}}(s)=g^{*}(s)=s / \theta^{*}$.)

The scheme of the proof of the properties (a) and (b) is the following.

Let us take the function $\widetilde{V}(x, s)$ defined by the right-hand side of (18). This function has two continuous first derivatives $\frac{\partial \widetilde{V}}{\partial x}$ and $\frac{\partial \widetilde{V}}{\partial s}$. The second derivative $\frac{\partial^{2} \widetilde{V}}{\partial x^{2}}$ is also continuous in $(x, s)$ except for the points on the line $x=s / \tilde{\theta}, s>0$. Hence, for each $s$, the function $\widetilde{V}(x, s)$ has continuous second derivative in $x$ except at the point $x=s / \tilde{\theta}$.

For $x<s / \tilde{\theta}$, the function $\tilde{V}(x, s)=s$, and for $x>s / \tilde{\theta}$ this function has a positive derivative $\frac{\partial^{2} \widetilde{V}}{\partial x^{2}}$. So, this function is convex (in $x$ ). This property allows us to apply the Ito-Meyer formula (see, for example, [5, Chapter V] or $\left[6\right.$, Chapter IV]) to the process $\left(e^{-(\lambda+r) t} \widetilde{V}\left(X_{t}, S_{t}\right)\right)_{t \geq 0}$ which gives (with left-side version of the second derivatives in the area $D(\tilde{\theta})$ )

$$
\begin{aligned}
e^{-(\lambda+r) t} & \widetilde{V}\left(X_{t}, S_{t}\right)=\widetilde{V}(x, s) \\
& +\int_{0}^{t} e^{-(\lambda+r) u}\left[L_{X} \widetilde{V}\left(X_{u}, S_{u}\right)-(\lambda+r) \widetilde{V}\left(X_{u}, S_{u}\right)\right] d u \\
& +\int_{0}^{t} e^{-(\lambda+r) u} \frac{\partial \widetilde{V}}{\partial s} d S_{u}+\int_{0}^{t} e^{-(\lambda+r) u} \sigma X_{u} \frac{\partial \widetilde{V}}{\partial x} d B_{u} .
\end{aligned}
$$


Observe that in (35) the integral on $d S_{u}$ is equal to zero because on the diagonal $\{(x, s) \in E: x=s\}$, where the process $S$ can only increase, the derivative $\frac{\partial \widetilde{V}}{\partial s}=0$. In the area $C(\tilde{\theta})$ of "continuation" of observation, we have

$$
L_{X} \widetilde{V}(x, s)-(\lambda+r) \widetilde{V}(x, s)=0,
$$

and in the area $D(\tilde{\theta})$ of "stopping", we have

$$
L_{X} \widetilde{V}(x, s)-(\lambda+r) \widetilde{V}(x, s) \leq 0 .
$$

Denoting by $\widetilde{M}_{\tau}$ the last integral in (35) we find from (35)-(37) that $\left(\mathbf{P}_{x, s^{-}}\right.$a.s. $)$

$$
e^{-(\lambda+r) t} \widetilde{V}\left(X_{t}, S_{t}\right) \leq \widetilde{V}(x, s)+\widetilde{M}_{t}
$$

The process $\widetilde{M}=\left(\widetilde{M}_{t}\right)_{t \geq 0}$ is a local martingale. If $\left(\sigma_{n}\right)_{n \geq 1}$ is a localizing sequence and $\tau$ is a stopping time of $\mathcal{M}$ then

$$
\mathbf{E}_{x, s} e^{-(\lambda+r)\left(\tau \wedge \sigma_{n}\right)} \widetilde{V}\left(X_{\tau \wedge \sigma_{n}}, S_{\tau \wedge \sigma_{n}}\right) \leq \widetilde{V}(x, s)
$$

because $\mathbf{E}_{x, s} \widetilde{M}_{\tau \wedge \sigma_{n}}=0$. We have also $s \leq \widetilde{V}(x, s)$. Therefore, from (39)

$$
\mathbf{E}_{x, s} e^{-(\lambda+r)\left(\tau \wedge \sigma_{n}\right)} S_{\tau \wedge \sigma_{n}} \leq \widetilde{V}(x, s) .
$$

Taking $n \rightarrow \infty$ and applying Fatou's lemma to (40) we find that

$$
\mathbf{E}_{x, s} e^{-(\lambda+r) \tau} S_{\tau} \leq \widetilde{V}(x, s)
$$

and hence $V^{*}(x, s) \leq \widetilde{V}(x, s)$. Now let us prove the validity of (34). In [1] it was shown that the stopping time $\tilde{\tau}\left(=\tau^{*}\right) \in \mathcal{M}$. Also from [1] it follows that the family $\left\{\widetilde{M}_{t \wedge \tilde{\tau}}, t \geq 0\right\}$ is uniformly integrable. This together with property $E_{x, s} \widetilde{M}_{t \wedge \sigma_{n} \wedge \tilde{\tau}}=0$ gives (when $t \rightarrow \infty, n \rightarrow \infty$ ) that $\mathbf{E}_{x, s} \widetilde{M}_{\tilde{\tau}}=0$. Taking into account that in the area $C(\theta)$ of continuation of observations, equality (36) does hold we get

$$
\mathbf{E}_{x, s} e^{-(\lambda+r) \tilde{\tau}} \tilde{V}\left(X_{\tilde{\tau}}, S_{\tilde{\tau}}\right)=\tilde{V}(x, s) .
$$

But $\tilde{V}\left(X_{\tilde{\tau}}, S_{\tilde{\tau}}\right)=S_{\tilde{\tau}}$. Therefore, equality (34) holds. This completes the proof of the theorem. 


\section{Russian Put option with Barrier}

Described in Section 1, the barrier version has a simple financial and economical interpretation being also of interest for other models. For our case we give explicit solution which shows some new effects which can appear in similar problems. We denote by

$$
\mathcal{M}_{\varepsilon}=\left\{\tau: \tau \leq T_{\varepsilon}\right\}
$$

the class of the stopping times less or equal to $T_{\varepsilon}=\inf \left\{t \geq 0: X_{t} \leq \varepsilon\right\}$, $\varepsilon>0$, that is, the class of first times when the process $X$ reaches the barrier on the level $\varepsilon$. We put

$$
V_{\varepsilon}^{*}(x, s)=\sup _{\tau \in \mathcal{M} \varepsilon} \mathbf{E}_{x, s} e^{-(\lambda+r) \tau} S_{\tau}
$$

It is clear that $V_{\varepsilon}^{*}(x, s)$ can be defined also as

$$
V_{\varepsilon}^{*}(x, s)=\sup _{\tau \in \mathcal{M}} \mathbf{E}_{x, s} e^{-(\lambda+r) \tau} S_{\tau} I\left(\tau \leq T_{\varepsilon}\right) .
$$

From these formulae

$$
V_{\varepsilon}^{*}(x, s) \leq V^{*}(x, s)
$$

From a financial point of view, the event $\left\{X_{t}<\varepsilon\right\}$ may be interpreted as a state of a default in time $t$. Hence, if we take $\tau$ in $\mathcal{M}_{\varepsilon}$, it means that a decision on stopping should be taken before time of default. Since $T_{\varepsilon}=\inf \left\{t: X_{t} \leq \varepsilon\right\}$, it is clear that for all $0<x<\varepsilon$, the optimal stopping time is $\tau_{\varepsilon}^{*}=0$ and in this (noninteresting) case, $V_{\varepsilon}^{*}(x, s)=s$ for all $s \geq x$. For this reason in the sequel we assume that $(x, s) \in E_{\varepsilon}$, where

$$
E_{\varepsilon}=\{(x, s) \in E: \varepsilon \leq x \leq s\}
$$

Put

$$
g_{\varepsilon}^{*}(s)=\max \left(\varepsilon, s / \theta^{*}\right),
$$

and define the following four sets (see Figure 2)

$$
\begin{array}{r}
D_{\mathrm{I}}^{*}:=\left\{(x, s) \in E_{\varepsilon}: x=\varepsilon, \varepsilon \leq s \leq \varepsilon \theta^{*}\right\}, \\
D_{\mathrm{II}}^{*}:=\left\{(x, s) \in E_{\varepsilon}: \varepsilon \leq x \leq s / \theta^{*}, s>\varepsilon \theta^{*}\right\}
\end{array}
$$


and

$$
\begin{array}{r}
C_{\mathrm{I}}^{*}:=\left\{(x, s) \in E_{\varepsilon}: \varepsilon<x \leq s, \varepsilon<s<\varepsilon \theta^{*}\right\}, \\
C_{\mathrm{II}}^{*}:=\left\{(x, s) \in E_{\varepsilon}: s / \theta^{*}<x \leq s, s \geq \varepsilon \theta^{*}\right\} .
\end{array}
$$

Theorem 3.1 An optimal stopping time for problem (41) (for $(x, s) \in E_{\varepsilon}$ ) is

$$
\tau_{\varepsilon}^{*}=\inf \left\{t: X_{t} \leq g_{\varepsilon}^{*}\left(S_{t}\right)\right\}
$$

or, equivalently,

$$
\tau_{\varepsilon}^{*}=\inf \left\{t:\left(X_{t}, S_{t}\right) \in D_{\mathrm{I}}^{*} \cup D_{\mathrm{II}}^{*}\right\} .
$$

Moreover, the value function (41) is given by

$$
V_{\varepsilon}^{*}(x, s)= \begin{cases}U_{\varepsilon}(x, s), & (x, s) \in C_{\mathrm{I}}^{*} \cup D_{\mathrm{I}}^{*}, \\ V^{*}(x, s), & (x, s) \in C_{\mathrm{II}}^{*} \cup D_{\mathrm{II}}^{*}\end{cases}
$$

where $V^{*}$ is given in (18) and

$$
\begin{gathered}
U_{\varepsilon}(x, s)=\left(\frac{x}{\varepsilon}\right)^{\gamma_{1}}\left\{-\frac{\varepsilon}{\beta}\left[\int_{\beta \log (s / \varepsilon)}^{\beta \log \theta^{*}} \frac{e^{(y+1) t}}{e^{t}-1} d t+\theta^{*} \gamma_{2}\right]\right\} \\
+\left(\frac{x}{\varepsilon}\right)^{\gamma_{2}}\left\{\frac{\varepsilon}{\beta}\left[\int_{\beta \log (s / \varepsilon)}^{\beta \log \theta^{*}} \frac{e^{y t}}{e^{t}-1} d t+\theta^{*} \gamma_{1}\right]\right\} .
\end{gathered}
$$

with

$$
\beta=\gamma_{2}-\gamma_{1}, \text { and } y=\beta^{-1}
$$

Proof : In the area $D^{*}:=D_{\mathrm{I}}^{*} \cup D_{\mathrm{II}}^{*}$, we have $V_{\varepsilon}(x, s)=s$ and $\tau_{\varepsilon}^{*}=0$. It is clear that if $x \geq \varepsilon$ and $s \geq \varepsilon \theta^{*}$, the optimal time $\tau^{*}$ (for problem (14)) satisfies the property $\tau^{*} \leq T_{\varepsilon}$, that is, $\tau^{*} \in \mathcal{M}_{\varepsilon}$, and because $V_{\varepsilon}^{*}(x, s) \leq V^{*}(x, s)$ we see that the optimal time $\tau_{\varepsilon}^{*}$ in $\mathcal{M}_{\varepsilon}$ can be taken equal to $\tau^{*}$. Hence, if $(x, s) \in C_{\mathrm{II}}^{*}$ then $\tau_{\varepsilon}^{*}=\tau^{*}$.

Consider now on the "triangular" area

$C_{\mathrm{I}}^{*} \cup D_{\mathrm{I}}^{*}=\left\{(x, s): \varepsilon<x<\varepsilon \theta^{*}, \varepsilon<s<\varepsilon \theta^{*}\right\} \bigcup\left\{(x, s): x=\varepsilon, \varepsilon \leq s<\varepsilon \theta^{*}\right\}$. 


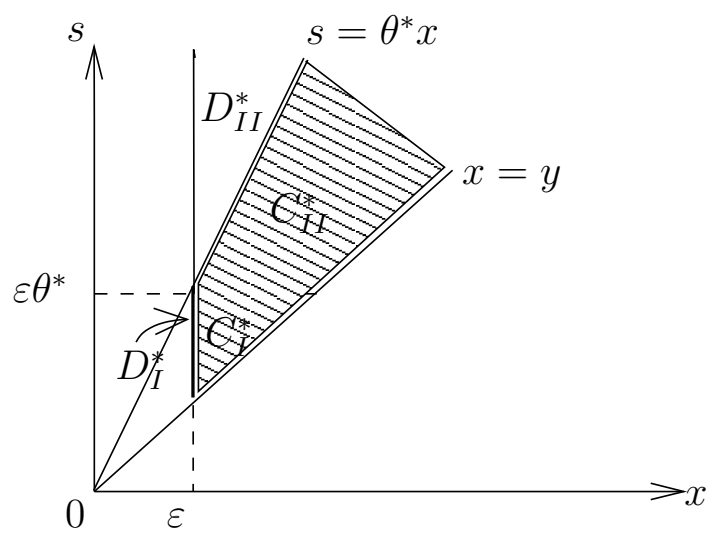

Figure 2: Optimal stopping area and continuation region for the Russian put option with barrier

If the random walk $\left(X_{t}, S_{t}\right)_{t \geq 0}$ starts in a point $(x, s) \in C_{\mathrm{I}}^{*}$, then there are two possibilities: 1 ) the random walk is terminated (in time $T_{\varepsilon}$ ) on the boundary $D_{\mathrm{I}}^{*}$; or 2$)$ after reaching the point $\left(\varepsilon \theta^{*}, \varepsilon \theta^{*}\right)$ and penetrating in the area $C_{\mathrm{II}}^{*}$, it will be terminated when the random walk reaches the stopping boundary $\left\{(x, s): x=s / \theta^{*}\right\} \cap E_{\varepsilon}$ separating areas $D_{\mathrm{II}}^{*}$ and $C_{\mathrm{II}}^{*}$. If $(x, s) \in D_{\mathrm{I}}^{*}$, then $T_{\varepsilon}=0$ and for such points the stopping time $\tau_{\varepsilon}^{*}=0$ is optimal in $\mathcal{M}_{\varepsilon}$. Consider now more carefully the case when the initial point $(x, s) \in C_{\mathrm{I}}^{*}$. Let

$$
\tau_{\left(\varepsilon \theta^{*}, \varepsilon \theta^{*}\right)}=\inf \left\{t \geq 0:\left(X_{t}, S_{t}\right)=\left(\varepsilon \theta^{*}, \varepsilon \theta^{*}\right)\right\}
$$

be the time of the first reaching of the "corner" point $\left(\varepsilon \theta^{*}, \varepsilon \theta^{*}\right)$. The event $\left\{T_{\varepsilon} \leq \tau_{\left(\varepsilon \theta^{*}, \varepsilon \theta^{*}\right)}\right\}$ means that the random walk leaves the "triangular" area through the boundary $D_{\mathrm{I}}^{*}$. The event $\left\{\tau_{\left(\varepsilon \theta^{*}, \varepsilon \theta^{*}\right)}<T_{\varepsilon}\right\}$ means that departure from $C_{\mathrm{I}}^{*}$ takes place through the "corner" point $\left(\varepsilon \theta^{*}, \varepsilon \theta^{*}\right)$ and then the time $\tau_{\varepsilon}^{*}$ will be the sum of the time $\tau_{\left(\varepsilon \theta^{*}, \varepsilon \theta^{*}\right)}$ and the time of exit from the point $\left(\varepsilon \theta^{*}, \varepsilon \theta^{*}\right)$ to the boundary $\left\{(x, s): x=s / \theta^{*}\right\} \cap E_{\varepsilon}$. These considerations show how to define the function $V_{\varepsilon}(x, s)=\mathbf{E}_{x, s} e^{-(\lambda+r) \tau_{\varepsilon}^{*}} S_{\tau_{\varepsilon}^{*}}$ for the points $(x, s)$ in $C_{\mathrm{I}}^{*}$.

Consider the following auxiliary problem: find the function $U_{\varepsilon}=U_{\varepsilon}(x, s)$ such that

$$
U_{\varepsilon}(x, s)=s, \quad \text { if } x=s, \varepsilon<s<\varepsilon \theta^{*},
$$




$$
\begin{gathered}
L_{X} U_{\varepsilon}(x, s)=(\lambda+r) U_{\varepsilon}(x, s), \quad \text { if } \varepsilon<x<\varepsilon \theta^{*}, \\
\lim _{x \uparrow s} \frac{\partial U_{\varepsilon}}{\partial s}(x, s)=0, \quad \text { if } \varepsilon<x<\varepsilon \theta^{*} .
\end{gathered}
$$

Additionally to (52)-(54), we consider also the condition

$$
U_{\varepsilon}(x, s)=V^{*}(x, s), \quad \text { if } \varepsilon \leq x \leq \theta^{*}, \quad s=\varepsilon \theta^{*},
$$

where $V^{*}(x, s)$ is the function defined by (18).

The general solution to (53) can be written as (see (28))

$$
U_{\varepsilon}(x, s)=A_{1}(s)\left(\frac{x}{\varepsilon}\right)^{\gamma_{1}}+A_{2}(s)\left(\frac{x}{\varepsilon}\right)^{\gamma_{2}} .
$$

To find $A_{1}(s)$ and $A_{2}(s)$, we use conditions (52) and (54). Then, assuming that $A_{1}(s)$ and $A_{2}(s)$ are differentiable we get

$$
\begin{gathered}
A_{1}(s)+A_{2}(s)=s \\
A_{1}^{\prime}(s)\left(\frac{s}{\varepsilon}\right)^{\gamma_{1}}+A_{2}^{\prime}(s)\left(\frac{s}{\varepsilon}\right)^{\gamma_{2}}=0 .
\end{gathered}
$$

From (57), we get

$$
A_{1}^{\prime}(s)+A_{2}^{\prime}(s)=1 .
$$

Taking into account (58), we find that

$$
\begin{aligned}
& A_{1}^{\prime}(s)=\frac{(s / \varepsilon)^{\gamma_{2}}}{(s / \varepsilon)^{\gamma_{2}}-(s / \varepsilon)^{\gamma_{1}}}=\frac{1}{1-(s / \varepsilon)^{\gamma_{1}-\gamma_{2}}}, \\
& A_{2}^{\prime}(s)=\frac{(s / \varepsilon)^{\gamma_{1}}}{(s / \varepsilon)^{\gamma_{1}}-(s / \varepsilon)^{\gamma_{2}}}=\frac{1}{1-(s / \varepsilon)^{\gamma_{2}-\gamma_{1}}},
\end{aligned}
$$

from which we get

$$
\begin{aligned}
A_{2}(s) & =-\int_{s}^{s \theta^{*}} \frac{d u}{1-(u / \varepsilon)^{\gamma_{2}-\gamma_{1}}}+C_{2} \\
& =\frac{\varepsilon}{\beta} \int_{\beta \log (s / \varepsilon)}^{\beta \log \theta^{*}} \frac{e^{y t}}{e^{t}-1} d t+C_{2},
\end{aligned}
$$


where $\beta$ and $y$ are defined in (51). Similarly,

$$
A_{1}(s)=-\frac{\varepsilon}{\beta} \int_{\beta \log (s / \varepsilon)}^{\beta \log \theta^{*}} \frac{e^{(y+1) t}}{e^{t}-1} d t+C_{1} .
$$

Now we must find the constants $C_{1}$ and $C_{2}$. We remark that in the point $(x, s)=\left(\varepsilon, \varepsilon \theta^{*}\right)$, we have

$$
A_{1}\left(\varepsilon \theta^{*}\right)+A_{2}\left(\varepsilon \theta^{*}\right)=\varepsilon \theta^{*}
$$

Thus,

$$
C_{1}+C_{2}=\varepsilon \theta^{*}
$$

In the point $(x, s)=\left(\varepsilon \theta^{*}, \varepsilon \theta^{*}\right)$, the equality $U(x, s)=V^{*}(x, s)$ holds and together with (18) and (56) leads to the relationship

$$
A_{1}\left(\varepsilon \theta^{*}\right)\left(\theta^{*}\right)^{\gamma_{1}}+A_{2}\left(\varepsilon \theta^{*}\right)\left(\theta^{*}\right)^{\gamma_{2}}=\frac{\varepsilon \theta^{*}}{\gamma_{2}-\gamma_{1}}\left[\gamma_{2}\left(\theta^{*}\right)^{\gamma_{1}}-\gamma_{1}\left(\theta^{*}\right)^{\gamma_{2}}\right] .
$$

Together with (61), (62) it gives

$$
C_{1}\left(\theta^{*}\right)^{\gamma_{1}}+C_{2}\left(\theta^{*}\right)^{\gamma_{2}}=\frac{\varepsilon \theta^{*}}{\gamma_{2}-\gamma_{1}}\left[\gamma_{2}\left(\theta^{*}\right)^{\gamma_{1}}-\gamma_{1}\left(\theta^{*}\right)^{\gamma_{2}}\right]
$$

Combining with (64), we obtain

$$
C_{1}=\frac{\varepsilon \theta^{*} \gamma_{2}}{\gamma_{2}-\gamma_{1}}, \quad C_{2}=\frac{\varepsilon \theta^{*} \gamma_{1}}{\gamma_{2}-\gamma_{1}} .
$$

Finally,

$$
\begin{gathered}
A_{1}(s)=-\frac{\varepsilon}{\beta}\left[\int_{\beta \log (s / \varepsilon)}^{\beta \log \theta^{*}} \frac{e^{(y+1) t}}{e^{t}-1} d t+\theta^{*} \gamma_{2}\right] \\
A_{2}(s)=\frac{\varepsilon}{\beta}\left[\int_{\beta \log (s / \varepsilon)}^{\beta \log \theta^{*}} \frac{e^{y t}}{e^{t}-1} d t+\theta^{*} \gamma_{1}\right]
\end{gathered}
$$

With these values we find that the solution of the problem $(52)-(54)$ for $(x, s)$ in $C_{\mathrm{I}}^{*}$ is given by $(50)$. 
From previous considerations it is easy to see, using the strong Markov property of the process $\left(X_{t}, S_{t}\right)_{t \geq 0}$, that the function

$$
V_{\varepsilon}(x, s)=\mathbf{E}_{x, s} e^{-(\lambda+r) \tau_{\varepsilon}^{*}} S_{\tau_{\varepsilon}^{*}}
$$

in the area $E_{\varepsilon}$ is given by the following expression:

$$
V_{\varepsilon}(x, s)= \begin{cases}U_{\varepsilon}(x, s), & (x, s) \in C_{\mathrm{I}}^{*} \cup D_{\mathrm{I}}^{*} \\ V^{*}(x, s), & (x, s) \in C_{\mathrm{II}}^{*} \cup D_{\mathrm{II}}^{*}\end{cases}
$$

We claim that $V_{\varepsilon}(x, s)=V_{\varepsilon}^{*}(x, s)$ and $\tau_{\varepsilon}^{*}$ is an optimal stopping time in the class $\mathcal{M}_{\varepsilon}$. The idea of the proof is the same as in Theorem 2.1 and is based on the verification of the properties (a) and (b) (see (32),(34)), which requires the use of the Ito-Meyer formula.

For $(x, s) \in C_{\mathrm{II}}^{*}$, we saw already that $\tau_{\varepsilon}^{*}=\tau^{*}$. Consequently, one need to consider only the case when $(x, s) \in C_{\mathrm{I}}^{*} \cup D_{\mathrm{I}}^{*}$. If $(x, s) \in D_{\mathrm{I}}^{*}$, then $T_{\varepsilon}=0$ and $\tau_{\varepsilon}^{*}=0$.

Now we apply the Ito-Meyer formula to the function $V_{\varepsilon}(x, s)$, where $(x, s) \in C_{\mathrm{I}}^{*}$. As in $(35)$

$$
\begin{aligned}
& e^{-(\lambda+r) t} V_{\varepsilon}\left(X_{t}, S_{t}\right)=V_{\varepsilon}(x, s)+\int_{0}^{t} e^{-(\lambda+r) u}\left[L_{X} V_{\varepsilon}\left(X_{u}, S_{u}\right)-(\lambda+r) V_{\varepsilon}\left(X_{u}, S_{u}\right)\right] d u \\
&+\int_{0}^{t} e^{-(\lambda+r) u} \frac{\partial V_{\varepsilon}}{\partial s} d S_{u}+\int_{0}^{t} e^{-(\lambda+r) u} \sigma X_{u} \frac{\partial V_{\varepsilon}}{\partial x}\left(X_{u}, S_{u}\right) d B_{u}
\end{aligned}
$$

Again the integral on $d S_{u}$ is equal to zero by condition (54). We have

$$
L_{X} V_{\varepsilon}\left(X_{u}, S_{u}\right)-(\lambda+r) V_{\varepsilon}\left(X_{u}, S_{u}\right) \leq 0 \quad \mathbf{P}_{x, s^{-}} \text {a.s. },(x, s) \in C_{\mathrm{I}}^{*} .
$$

Hence, as in (38)

$$
e^{-(\lambda+r) t} V_{\varepsilon}\left(X_{t}, S_{t}\right) \leq V_{\varepsilon}(x, s)+M_{t},
$$

where $M_{t}$ is the local martingale

$$
M_{t}=\int_{0}^{t} e^{-(\lambda+r) u} \sigma X_{u} \frac{\partial V_{\varepsilon}}{\partial x}\left(X_{u}, S_{u}\right) d B_{u} .
$$

From (71) we conclude, as in the end of the proof of Theorem 2.1, that for any stopping time $\tau \in \mathcal{M}_{\varepsilon}$,

$$
\mathbf{E}_{x, s} e^{(-\lambda+r) \tau} S_{\tau} \leq V_{\varepsilon}(x, s) .
$$


Consequently, $V_{\varepsilon}^{*}(x, s) \leq V_{\varepsilon}(x, s)$.

As in [1], we may check that the family $\left\{M_{t \wedge \tau_{\varepsilon}^{*}}, t \geq 0\right\}$ is uniformly integrable. This property together with the property that

$$
L_{X} V_{\varepsilon}(x, s)-(\lambda+r) V_{\varepsilon}(x, s)=0
$$

for all points $(x, s) \in C_{\mathrm{I}}^{*} \cup C_{\mathrm{II}}^{*}$, leads to

$$
\mathbf{E}_{x, s} e^{(-\lambda+r) \tau_{\varepsilon}^{*}} V_{\varepsilon}\left(X_{\tau_{\varepsilon}^{*}}, S_{\tau_{\varepsilon}^{*}}\right) \leq V_{\varepsilon}(x, s) .
$$

With $\mathbf{P}_{x, s}$-probability one, $V_{\varepsilon}\left(X_{\tau_{\varepsilon}^{*}}, S_{\tau_{\varepsilon}^{*}}\right)=S_{\tau_{\varepsilon}^{*}}$ for all $(x, s) \in E_{\varepsilon}$. Therefore, the stopping time $\tau_{\varepsilon}^{*}$ is optimal for problem (41). This completes the proof of Theorem 3.1.

\section{References}

[1] L. A. Shepp, A. N. Shiryaev. The Russian option: Reduced regret. Ann. Appl. Probab. 1993. V. 3. No. 3. P. 631-640.

[2] L. A. Shepp, A. N. Shiryaev. A new look at the "Russian option". Theory Probab. Appl. 1994. V. 39. No. 1. P. 103-119.

[3] L. A. Shepp, A. N. Shiryaev. A dual Russian option for selling short. Probability Theory and Mathematical Statistics: Lectures presented at the semester held in St. Peterburg, Russia, March 2 - April 23, 1993. Ed. by I. A. Ibragimov et al. Amsterdam: Gordon \& Breach, 1996, 209-218.

[4] A. N. Shiryaev. Essentials of Stochastic Finance. Singapore: World Scientific, 1999.

[5] J. Jacod. Calcul Stochastique et Problèmes de Martingales. Berlin etc.: Springer-Verlag, 1979. (Lecture Notes in Math. V. 714.)

[6] Ph, Protter. Stochastic Integration and Differential Equations: A new approach. Berlin etc.: Springer-Verlag, 1990. (Appl. Math. V. 21.) 